\section{A Case series on Asthma-COPD overlap (ACO) is independent from other chronic obstructive diseases (COPD and Asthma)}

\section{Divya Khanduja ${ }^{1 *}$ and Naveen Pandhi ${ }^{2}$}

\author{
1Junior Resident, Department of Pulmonary Medicine, Government Medical College, Amritsar, \\ Punjab, India \\ ${ }^{2}$ Professor and Head, Department of Pulmonary Medicine, Government Medical College, Amritsar, \\ Punjab, India
}

\section{Abstract}

As we know that, Asthma and chronic obstructive pulmonary diseases are well characterized diseases, they can co-exist as asthma-COPD overlap (ACO). The co-existence of asthma-chronic obstructive pulmonary disease overlap (ACO) in chronic obstructive pulmonary disease (COPD) patients is often unrecognized. In patients with a primary diagnosis of COPD or Asthma, the identification of ACO has got implication for better prognosis and treatment. Such patients experience frequent exacerbations, poor quality of life, rapid decline in lung function and high mortality than COPD or Asthma alone. Inhalational steroids provide significant alleviation of symptoms in such patients and some studies suggest that the most severe patients may respond to biological agents indicated for severe asthma. Patients who have asthma with a COPD component tend to present with severe hypoxia because of Irreversible/fixed airway obstruction and impairment of the alveolar diffusion capacity by emphysematous changes. In contrast, patients with COPD who have an asthma component not only have exertional dyspnoea but also develop paroxysmal wheezing or dyspnoea at night or in the early morning. The criteria to diagnose asthma-COPD overlap (ACO) include positive bronchodilator response, sputum eosinophilia or previous diagnosis of asthma, high IgE and/or history of atopy. There is scarcity of literature available in country like India. We highlight the importance of identification of Asthma COPD overlap as different phenotype from COPD or asthma alone as it is challenging to diagnose ACO in India. We report 3 cases having both the features of asthma and COPD, later diagnosed with Asthma-COPD overlap.

\section{More Information}

*Address for Correspondence: Divya Khanduja Junior Resident, Department of Pulmonary Medicine, Government Medical College, Amritsar, Punjab, India, Tel: 9582969172; Email: divyakhandujalhmc@gmail.com

Submitted: July 06, 2021

Approved: July 29, 2021

Published: July 30, 2021

How to cite this article: Khanduja D, Pandhi N. A Case series on Asthma-COPD overlap (ACO) is independent from other chronic obstructive diseases (COPD and Asthma). J Pulmonol Respir Res. 2021; 5: 054-058.

DOI: 10.29328/journal.jprr.1001025

Copyright: ๑ 2021 Khanduja D, et al. This is an open access article distributed under the Creative Commons Attribution License, which permits unrestricted use, distribution, and reproduction in any medium, provided the original work is properly cited. Keywords: Asthma; COPD; Asthma-COPD
overlap; Eosinophilia; ACO

Abbreviations: ACO: Asthma-COPD Overlap; COPD: Chronic Obstructive Pulmonary Disease; GINA: Global Initiative for Asthma; GOLD: Global Initiative for Chronic Obstructive Lung Disease; AEC: Absolute Eosinophil Count; ICS: Inhalational Corticosteroids; LABA: Long-Acting Beta Agonists; LAMA: Long-Acting Muscarinic Antagonist

Check for updates

OPEn ACCESS

\section{Introduction}

The global burdens of asthma and chronic obstructive pulmonary disease (COPD) are increasing, each of which was estimated to affect respectively approximate 339 million and 251 million people worldwide in 2016 [1]. Asthma is a heterogeneous disease, usually characterized by chronic airway inflammation. It is defined by the history of respiratory symptoms such as wheeze, shortness of breath, chest tightness, and cough that vary over time and in intensity, together with variable expiratory airflow limitation [2]. Chronic obstructive pulmonary disease (COPD) is a common, preventable and treatable disease that is characterized by persistent respiratory symptoms and airflow limitation that is due to airway and/or alveolar abnormalities usually caused by significant exposure to noxious particles or gases [3]. Although, Chronic obstructive pulmonary disease (COPD) and asthma are different entities but, in some patients, they can share features of both the diseases. "Asthma-COPD overlap (ACO)" as descriptive term was used to define these patients. ACO prevalence has varied widely in studies: from $0.9 \%$ to $11.1 \%$ in the general population, from $11.1 \%$ to $61.0 \%$ in asthma patients, and from $4.2 \%$ to $66.0 \%$ in COPD patients [4]. There is little data available on ACO in India. In 2015, the Global Initiative for Asthma (GINA) and the Global Initiative for Chronic Obstructive Lung Disease (GOLD) released a joint statement which was then called "asthma COPD overlap syndrome (ACOS)", as persistent airflow limitation with several features usually associated with asthma and several features usually associated with COPD [5]. In 2017, the American Thoracic Society and the National Heart, Lung and Blood Institute published a joint workshop report on ACO. It was concluded that ACO, like asthma and COPD, doesn't represent a single discrete disease entity [6]. Thus, it is important to diagnose the patients with $\mathrm{ACO}$ as they have higher chances of exacerbations which are more severe than 
as compared with patients of asthma or COPD alone. Herein we report a case of ACO sharing clinical features of both asthma and COPD.

\section{Case presentation 1}

A 61-year-old male presented at emergency with complaints of nocturnal and early morning shortness of breath, cough with expectoration and wheezing episodes for past 2 weeks. He gave history of similar episodes for past 6-7 years. There is no history of breathlessness in childhood. Patient was diagnosed with COPD 6 years back. There is history of progressive worsening of exertional dyspnoea, seasonal variation of breathlessness with symptoms on exposure to dust and were more during cold climate that often need hospitalization since past 6-7 years. He gave history of biomass fuel exposure since childhood, might be the causative factor in developing emphysematous changes in this case. He is non-smoker, non-alcoholic. There is no history of tuberculosis in past and family. On examination, patient was slightly dyspnoeic without the use of accessory muscles, with a blood pressure of $132 / 90 \mathrm{mmHg}$, pulse rate of $108 / \mathrm{min}$, respiratory rate of $34 / \mathrm{min}$, and saturation of $92 \%$ on room air. On general physical examination, clubbing was present and shape of the chest was barrel shaped. On auscultation, bilateral rhonchi with decreased breath sounds were present.

Laboratory findings revealed haemoglobin of $12.9 \mathrm{~g} / \mathrm{dl}$, white blood count of 10,600, ESR-28, RBS-104 mg/dl, serum bilirubin-0.41, SGOT-22, SGPT-19, serum creatinine-0.98. Peripheral eosinophilia was present (AEC-766). His sputum for acid fast bacilli, gram stain and culture and fungus were negative. Sputum for CBNAAT (Xpert MTB/RIF) showed MTB not detected. Mantoux test and serology of HIV were negative. We should rule out other conditions associated with eosinophilia such as parasitosis, allergic bronchopulmonary aspergillosis or Churg Strauss syndrome.

As we can see in figure 1, Chest radiograph showed bilateral hyperinflation with flattening of diaphragm and obliteration of right sided costophrenic angle. As the patient was not affordable, patient denied for HRCT chest which was advised to the patient.

As we can see in Figure 2 and Table 1, Spirometry showed: $\mathrm{FEV}_{1}-57 \%$, Post bronchodilator $\mathrm{FEV}_{1}-69 \%, \mathrm{FEV}_{1} / \mathrm{FVC}-59 \%$, Post Bronchodilator $\mathrm{FEV}_{1} / \mathrm{FVC}-61 \%$, Post bronchodilator increase in $\mathrm{FEV}_{1}-20 \%$.

Patient was on ICS and LABA combination for the treatment. Owing to these findings of reversibility of airflow limitation and peripheral eosinophilia. Patient was considered for the diagnosis of asthma-COPD overlap (ACO). Thus, patient started with the ICS, LABA and LAMA combination. After 4 week follow up, patient was clinically improved with no nocturnal dyspnoea and did not have wheezing with decrease in peripheral eosinophilia (AEC-225). Currently patient is following this pharmacological therapy and is clinically improved.

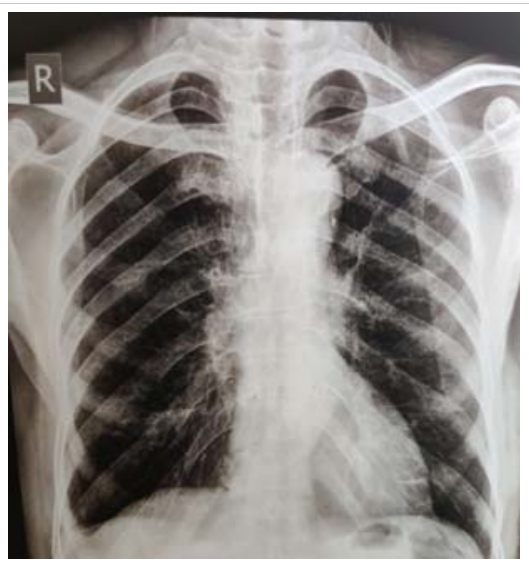

Figure 1: CXR PA View: Bilateral hyperinflation with flattening of diaphragm and obliteration of right sided costophrenic angle.

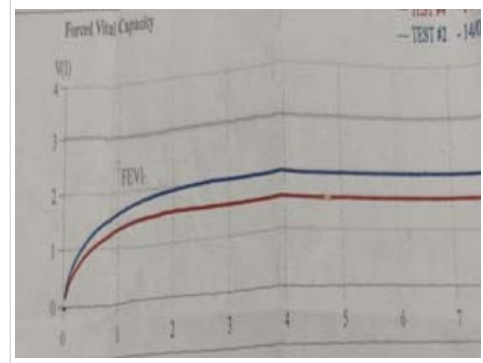

(a)

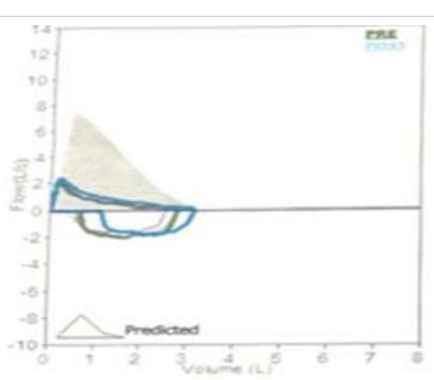

(b)
Figure 2: Showing (a) flow-volume curve and (b) volume-time curve of spirometry

\begin{tabular}{|c|c|c|}
\hline \multicolumn{1}{|c|}{ Table 1: Spirometric interpretation of _figure 2. } & \\
\hline Pre FEV & Litres & Percentage \\
\hline Post FEV $_{1}$ & 1.44 & 57 \\
\hline Pre FEV $_{1} /$ FVC & 1.74 & 69 \\
\hline Post FEV $/$ FVC & & 59 \\
\hline$\%$ Increase in FEV & & 61 \\
\hline
\end{tabular}

\section{Case presentation 2}

A 60-year-old male presented at emergency with complaints of fever, cough with expectoration for past 2 weeks and difficulty in breathing for past 2 days. He gave history of breathlessness since childhood from 15-years of age. He also gave history of seasonal variation in difficulty in breathing on exposure to dust and was more during winters that often require hospitalisation. He gave history of smoking for past 35 years with more than 10 pack years. He gave history of exertional dyspnoea for past 4 years which made it difficult to perform day to day activities. There was no history of tuberculosis in past and in family. On examination, patient was dyspnoeic, with blood pressure of $132 / 90$, pulse rate of $110 / \mathrm{min}$, respiratory rate of $32 / \mathrm{min}$ and saturation was $86 \%$ on room air. On general physical examination, pallor and clubbing was present. On auscultation, bilateral rhonchi with decreased breath sounds on infra-scapular areas was present.

Laboratory investigations revealed a white cell count of 14600, Haemoglobin of $13.3 \mathrm{~g} / \mathrm{dl}$, ESR-20 mm/hr, RBS-112 
$\mathrm{mg} / \mathrm{dl}$, serum bilirubin-0.54, SGOT-31, SGPT-26, ALP-0.90. Peripheral eosinophilia was present (AEC-628). His serum electrolytes and renal function test were within normal range. His sputum for acid fast bacilli and fungus were negative, sputum for gram stain and culture and sensitivity showed growth of pseudomonas. Sputum for CBNAAT (Xpert MTB/RIF) showed mycobacterium tuberculosis not detected. Mantoux test and serology of HIV, HCV and HBsAg were negative.

As we can see in Figure 3, Chest radiograph showed bilateral hyperinflation and flattening of diaphragm.

As we can see in Table 2, Spirometry showed $\mathrm{FEV}_{1-}$ $46 \%$, Post Bronchodilator $\mathrm{FEV}_{1}-53 \%, \mathrm{FEV}_{1} / \mathrm{FVC}-56 \%$, Post Bronchodilator $\mathrm{FEV}_{1} / \mathrm{FVC}-56 \%$, Post Bronchodilator increase in $\mathrm{FEV}_{1}-21 \%$.

Previously patient was on ICS and SABA combination as and when needed. But from the last 4 years patient was started with ICS and LABA combination. Then also the symptoms got worsened. With the high degree of suspicion, patient was diagnosed with ACO based upon clinical manifestations, peripheral eosinophilia and reversible airflow limitation. Patient was then started with ICS, LABA and LAMA combination. With subsequent follow up visits, patient showed improvement of symptoms and reduce frequency of exacerbations.

\section{Case presentation 3}

A 70-year-old male at the emergency with complaints of cough with expectoration for past 2 weeks and difficulty in breathing for past 4 days. He was diagnosed with COPD 4 years back. Patient had history of frequent exacerbations for past 2 years requiring hospital admission. He gave history of chronic rhinitis for past 2 years. There was no history of

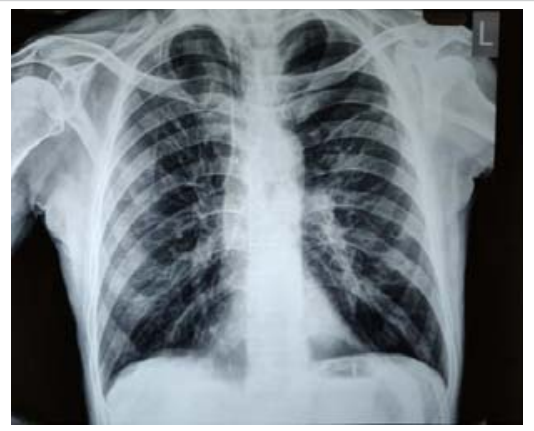

Figure 3: CXR-PA view showed bilateral hyperinflation and flattening of diaphragm.

\begin{tabular}{|c|c|c|}
\hline \multicolumn{1}{|l|}{ Table 2: Spirometry. } & Litres & \\
\hline Pre FEV $_{1}$ & 1.12 & 46 \\
\hline Post FEV $_{1}$ & 1.35 & 53 \\
\hline Pre $\mathrm{FEV}_{1} /$ FVC & & 56 \\
\hline Post $\mathrm{FEV}_{1} / \mathrm{FVC}$ & & 56 \\
\hline$\%$ Increase in FEV & & 21 \\
\hline
\end{tabular}

breathlessness in childhood. There was no history of asthma symptoms. He gave history of nocturnal dyspnoea, cough and wheezing episodes in past 2 years which often required hospitalization. There is no history of worsening of symptoms on exposure to dust or during cold climate. He gave history of smoking for past 50 years with 110 pack per year. There is no history of tuberculosis in past and family. On examination, patient was slightly dyspnoeic without the use of accessory muscles, with blood pressure of $140 / 90 \_\mathrm{mmHg}$, pulse rate of $112 / \mathrm{min}$, respiratory rate of $33 / \mathrm{min}$, and saturation of $90 \%$ on room air. On general physical examination, clubbing was present and shape of the chest was barrel shaped. On auscultation, bilateral rhonchi with decreased breath sounds were present.

Laboratory findings revealed Haemoglobin of $13.1 \mathrm{~g} / \mathrm{dl}$, white blood count of 11,4oo, ESR-18, RBS-112 mg/dl, serum bilirubin-0.5, SGOT-28, SGPT-21, serum creatinine-0.6. Peripheral eosinophilia was present (AEC-924). His sputum for acid fast bacilli, gram stain and culture and fungus were negative. Sputum for CBNAAT (Xpert MTB/RIF) showed MTB not detected. Mantoux test and serology of HIV were negative. We should rule out other conditions associated with eosinophilia such as parasitosis, allergic bronchopulmonary aspergillosis or Churg Strauss syndrome.

As we can see in Figure 4, CT chest showed areas of decreased attenuation and emphysematous changes and thickening of the septa.

As we can see in Table 3, Spirometry showed $\mathrm{FEV}_{1}$ $74 \%$, Post Bronchodilator $\mathrm{FEV}_{1}-86 \%, \mathrm{FEV}_{1} / \mathrm{FVC}-51 \%$, Post Bronchodilator $\mathrm{FEV}_{1} / \mathrm{FEV}-51 \%$, Post Bronchodilator increase in $\mathrm{FEV}_{1}-15 \%$.

Owing to the clinical manifestations, eosinophilia and

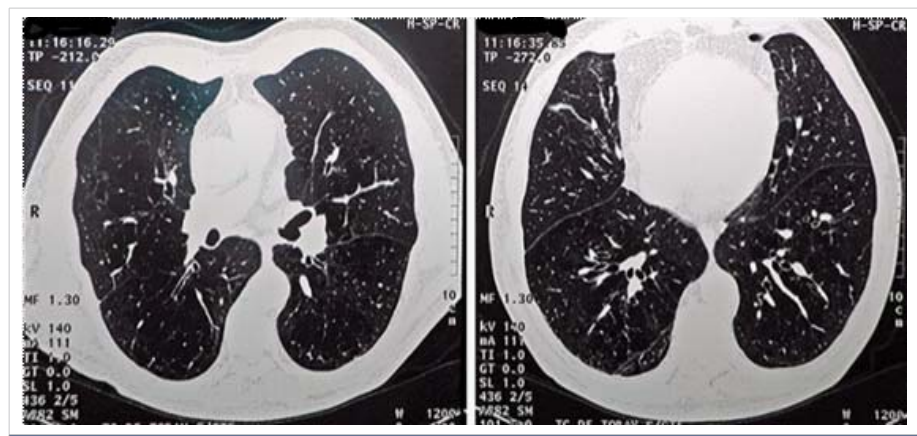

Figure 4: CT chest showed areas of decreased attenuation and emphysematous changes and thickening of the septa.

\begin{tabular}{|c|c|c|}
\hline \multicolumn{1}{|c|}{ Table 3: Spirometry. } & Percentage \\
\hline Pre FEV $_{1}$ & 2.11 & 74 \\
\hline Post FEV $_{1}$ & 2.44 & 86 \\
\hline Pre $\mathrm{FEV}_{1} /$ FVC & & 51 \\
\hline${\text { Post } \mathrm{FEV}_{1} / \mathrm{FVC}}$ & & 51 \\
\hline \% Increase in FEV & & 15 \\
\hline
\end{tabular}


reversibility of airflow, patient was diagnosed with ACO. Before the diagnosis, patient was on LAMA and LABA combination. After diagnosis patient was started on ICS, LABA and LAMA combination. With the subsequent follow up visits patient didn't show wheezing on examination and improvement in nocturnal symptoms.

\section{Discussion}

Asthma and Chronic obstructive pulmonary disease (COPD) are umbrella labels for heterogeneous conditions characterized by chronic airway and/or lung disease [2]. COPD and asthma each include several different clinical phenotypes, and are likely to have several different underlying mechanisms, some of which may be common to both asthma and COPD. The term 'asthma COPD overlap' (ACO) simply describes patients who features of both asthma and COPD [2]. In 1961, Orie and colleagues proposed the concept of overlap between COPD and asthma as all the airway diseases, including asthma, emphysema and chronic bronchitis, should be considered a single disease with common genetic origins which was subsequently known as "Dutch hypothesis" [7]. In 2015, the Global Initiative for Asthma (GINA) and the Global Initiative for Chronic Obstructive Lung Disease (GOLD) released a joint statement which was then called "asthma COPD overlap syndrome (ACOS)", as persistent airflow limitation with several features usually associated with asthma and several features usually associated with COPD [5]. Later, the word syndrome was removed as ACO have several different clinical manifestations and didn't fulfil the criteria to known as syndrome.

Asthma is a heterogenous and inflammatory disease affecting large and small respiratory tracts but not the lung parenchyma, and contains clusters of demographical, clinical and pathophysiological characteristics supported by different pathophysiological processes [8]. This heterogeneity may be explained by the complexity of dysregulated innate and adaptive inflammatory responses to exogenous allergens and proteases leading to the spectrum of abnormal tissue remodelling, where type 2 cytokines such as interleukin (IL)-4, IL-13 and IL-5 primarily promote airway eosinophilinfiltration, mucus hypersecretion, bronchial hyperresponsiveness and mast cell activation [9] . Major subpopulations of asthmatics have molecular signatures of T helper 2 (Th2)-inflammation and airway obstruction that markedly respond to inhaled corticosteroid (ICS) [10].

COPD is defined as a common, preventable and treatable disease that is characterized by persistent respiratory symptoms and airflow limitation that is due to airway and/or alveolar abnormalities usually caused by significant exposure to noxious particles or gases and influenced by host factors including abnormal lung development [3]. In addition to cigarette smoking, known as the most common COPD risk factor [11], the susceptibility could be influenced by genetic factors and abnormal lung growth. Unlike asthma, CD4+ T helper 1 (Th1) cells, CD8+ cytotoxic T (Tc) cells, neutrophils and macrophages predominantly affect the small airways and the lung parenchyma leading to mucus hypersecretion, alveolar wall destruction (emphysema) and small airway fibrosis in COPD. The small airway narrowing induced by pro-inflammatory cell infiltration, luminal exudates, wall thickening, and the loss of small airways associated with emphysema increases airway obstruction. The airflow limitation progressively leads to gas-trapping in peripheral lungs during expiration on exercise, resulting in dynamic hyperinflation which is postulated to be the main mechanism of exertional dyspnoea [12]. Thus bronchodilators, longacting muscarinic antagonists (LAMA) and long acting beta2agonists (LABA), are commonly used as the pharmacological therapy for COPD and are known to reduce lung hyperinflation, dyspnoea and exercise endurance [13]. Some COPD patients releases type 2 cytokines, may manifests clinical features as asthma such as bronchodilator reversibility, increase in peripheral eosinophilia and also in airways, these phenotypes thought to be associated to ACO [14].

In 2016, a global expert panel discussion between various specialists from North America, Western Europe and Asia, described a consensus on ACO which was based on major and minor clinical, spirometric and laboratory criteria [15]. According to this consensus, the patients who meet all the three major criteria and at least one minor criterion be considered for the diagnosis of ACO.

- Major criteria include [15]:

1. Persistent airflow limitation [post-bronchodilator $\mathrm{FEV}_{1} / \mathrm{FVC}<0.70$ ] in individuals 40 years of age or older.

2. At least 10 pack-years of tobacco smoking or equivalent indoor or outdoor air pollution exposure [ex. biomass].

3. History of asthma before 40 years of age or bronchodilator response of $>400 \mathrm{ml}$ in $\mathrm{FEV}_{1}$.

- Minor criteria include [15]:

1. History of atopy or allergic rhinitis.

2. Bronchodilator response of $\mathrm{FEV}_{1}>200 \mathrm{ml}$ and $12 \%$ from baseline values on 2 or more visits.

3. Peripheral blood eosinophil count of $>300$ cells/ microL.

Management of Asthma and COPD is different. For Asthma patients ICS is essential for treatment and for COPD patients LAMA and/or LABA should be given initially. ICS reduce serum IgE and sputum eosinophils, and improves lung function and patient-reported symptom scores in some patients with ACO. 
ICS should be given earlier in asthma patients and in COPD patients, ICS should be given with a history of more than and equal to 2 exacerbations requiring OCS and/or increased blood eosinophils (> 300/microL) [2,3]. Importantly, in asthma patients long-acting beta- 2 agonists (LABA) should be given in combination with ICS as LABA alone have been associated with an increased risk of asthma-related death when not given in combination with an ICS, thus LABA should not be prescribed as a single therapy in asthma [16]. For patients with ACO, ICS containing treatment is essential to reduce risk of severe exacerbations and death. LABA and/or LAMA usually needed as add-on [2]. Triple therapy (ICS+LABA+LAMA) has found to be more effective as comparison to ICS+LABA combination as it improves lung function and reduce the chances of exacerbations [17]. Therefore, LAMA should be added with ICS and LABA in ACO patients, if symptoms are not controlled with ICS and LABA combination to prevent risk of recurrent exacerbations. Non-pharmacological therapy such as pulmonary rehabilitation should be done in ACO patients with uncontrolled symptomatology (frequent exacerbations). Risk factors exposure control (smoking, biomass, allergens exposure) and vaccination coverage (influenza and antipneumococcal) should be considered.

\section{Conclusion}

ACO as a clinical entity have both the clinical features of asthma and COPD, it has been associated with increased disease burden and imposed challenges for diagnosis and management of these patients. According to the current definitions of asthma and COPD, ACO has been described as a subgroup of patients with several different clinical phenotypes reflecting different underlying mechanisms. Due to lack of data availability in India, it is challenging to diagnose ACO. This is important to recognise ACO in COPD or Asthma patients because an appropriate diagnostic measures for the ACO patients may allow better-targeted therapy, and helped to alleviate the worsening symptoms and recurrent exacerbations, thus, improved clinical course of the disease. This is much needed in COPD and asthma patients.

\section{References}

1. Vos T, Abajobir AA, Abate KH, Abbafati C, Abbas KM, et al. Global, regional, and national incidence, prevalence, and years lived with disability for 328 diseases and injuries for 195 countries, 1990-2016: A systematic analysis for the global burden of disease study 2016 Lancet. 2017; 390: 1211-1259.

PubMed: https://pubmed.ncbi.nlm.nih.gov/28919117/

2. Global Initiative for Asthma. Global strategy for asthma management and prevention; 2021. http://www.ginasthma.org.

3. Global Initiative for Chronic Obstructive Lung Disease (GOLD). Global strategy for the diagnosis, management and prevention of COPD. 2021 Report; 2021. http://www.goldcopd.org.

4. UchidaA, SakaueK, InoueH.Epidemiologyofasthma-chronicobstructive pulmonary disease overlap (ACO). Allergol Int. 2018; 67: 165-171. PubMed: https://pubmed.ncbi.nlm.nih.gov/29551279/

5. GINA-GOLD diagnosis of disease of chronic airflow limitation: asthma, COPD and asthma-COPD overlap syndrome (ACOS). 2020. https:// goldcopd.org/asthma-copd-asthma-copd-over lap-syndrome/

6. Woodruff PG, van den Berge M, Boucher RC, Brightling C, Burchard EG et al. American Thoracic Society/National Heart, Lung, and Blood Institute Asthma-Chronic Obstructive Pulmonary Disease Overlap Workshop Report. Am J Respir Crit Care Med. 2017; 196: 375-381. PubMed: https://pubmed.ncbi.nlm.nih.gov/28636425/

7. Orie NGM, Sluiter HJ, eds. Bronchitis. Assen, the Netherlands: Royal van Gorcum; 1962.

8. Wenzel SE. Asthma phenotypes: The evolution from clinical to molecular approaches. Nat Med. 2012; 18: 716-725.

PubMed: https://pubmed.ncbi.nlm.nih.gov/22561835/

9. Lambrecht BN, Hammad H, Fahy JV. The cytokines of asthma Immunity. 2019; 50: 975-991.

PubMed: https://pubmed.ncbi.nlm.nih.gov/30995510/

10. Woodruff PG, Modrek B, Choy DF, Jia G, Abbas AR, et al. T-Helper type 2-driven inflammation defines major subphenotypes of asthma. Am J Resp Crit Care Med. 2009; 180L 388-395.

PubMed: https://pubmed.ncbi.nlm.nih.gov/19483109/

11. Kohansal R, Martinez-Camblor P, Agustí A, Buist AS, Mannino DM, et al. The natural history of chronic airflow obstruction revisited. Am J Resp Crit Care Med. 2009; 180: 3-10.

PubMed: https://pubmed.ncbi.nlm.nih.gov/19342411/

12. Elbehairy AF, Ciavaglia CE, Webb KA, Guenette JA, Jensen D, et al Pulmonary gas exchange abnormalities in mild chronic obstructive pulmonary disease. implications for dyspnea and exercise intolerance. Am J Resp Crit Care Med. 2015; 191: 1384-1394.

PubMed: https://pubmed.ncbi.nlm.nih.gov/25826478/

13. O'Donnell DE, Flüge T, Gerken F, Hamilton A, Webb K, et al. Effects of tiotropium on lung hyperinflation, dyspnoea and exercise tolerance in COPD. Eur Respir J. 2004; 23: 832-840.

PubMed: https://pubmed.ncbi.nlm.nih.gov/15218994/

14. Christenson SA, Steiling K, van den Berge M, Hijazi K, Hiemstra PS, et al. Asthma-COPD overlap: clinical relevance of genomic signatures of type 2 inflammation in COPD. Am J Respir Crit Care Med. 2015; 191: 758-766. PubMed: https://pubmed.ncbi.nlm.nih.gov/25611785/

15. Sin DD, Miravitlles M, Mannino DM, Soriano JB, Price D, et al. What is asthma-COPD overlap syndrome (ACOS)? Towards a consensus definition from a roundtable discussion. Eur Respir J. 2016; 48: 664-673. PubMed: https://pubmed.ncbi.nlm.nih.gov/27338195/

16. Nelson HS, Weiss ST, Bleecker ER, Yancey SW, Dorinsky PM, et al. The Salmeterol Multicenter Asthma Research Trial: a comparison of usual pharmacotherapy for asthma or usual pharmacotherapy plus salmeterol. Chest. 2006; 129: 15-26.

PubMed: https://pubmed.ncbi.nlm.nih.gov/16424409/

17. Kew KM, Dahri K. Long-acting muscarinic antagonists (LAMA) added to combination long-acting beta2-agonists and inhaled corticosteroids (LABA/ICS) versus LABA/ICS for adults with asthma. Cochrane Database Syst Rev. 2016; 1: CD011721.

PubMed: https://pubmed.ncbi.nlm.nih.gov/26798035/ 